Journal of Teaching and Learning with Technology, Vol. 6, No. 1, January 2017, pp.76-80.

doi:10.14434/jotlt.v6n1.22367

\title{
Designing and Managing Engaging Discussions in Online Courses
}

\begin{abstract}
Micah Pollak ${ }^{1}$
Abstract: As 100\% online courses become more popular, the need for engaging student interactions through online discussions becomes more important. Unlike a traditional face-to-face course, where student-instructor and student-student interaction often naturally in the classroom, in an online class interaction needs to be consistently and deliberately promoted. As Velez-Solic (2015, p. 40) states, "Interaction is the heart of an online course." Extensive interaction is also the core of the social constructivism approach to education (Vygotsky, 1980). The use of weekly discussions surrounding a set of “discussion questions” is an increasingly popular way to achieve both student engagement and interaction. To enhance this interaction, I share two sets of tips for creating engaging discussions and effectively managing them in online courses. The first deals with developing discussion questions and the second focuses on the role of the instructor in moderating and evaluating student posts.
\end{abstract}

Keywords: teaching, online, discussions

To design effective and engaging discussion questions is not easy. Dillon (1983) argues that to conceive of an educative question requires thought, to formulate it requires labor and to pose it requires tact. To meet the challenges of creating engaging discussion questions, I designed the A.V.I.D. approach to question design, which stands for (A)ctive, (V)aried, (I)nteresting and openende(D). This approach assists with creating discussion questions that are likely to promote discussion and to be more engaging for students. The four parts of this acronym are:

\section{(A)ctive}

One of the common pitfalls when designing discussion questions is to choose those that lead to passive responses. Questions that begin with "What do you think about...” or "Apply what we've learned...” encourage students to treat discussion questions as a regurgitation of opinion. In an online course, students already spend much of their time sitting in front of their computer and discussions provide an opportunity to make the environment more dynamic. Whenever possible, I include at least some active elements to discussion questions, elements that may require them to reflect and return to the discussion later or physically get up from the computer. These active elements are similar to the "authentic activities" of Herrington, Oliver and Reeves (2002). They argue that activities which have real-world relevance and require students to investigate and define

\footnotetext{
${ }^{1}$ School of Business and Economics, Indiana University Northwest, 3400 Broadway, Gary, IN 46408, mpollak@iun.edu
} 
some tasks of the activity on their own are consistent with the constructivist educational philosophy and can be beneficial to the learner.

For example, in one discussion on the Supply \& Demand model I have students pick a good (such as "coffee") and then poll five friends on the "most they would be willing to pay" for the good. I then have them visit several stores and record the price they find of the good. When they return to the computer, they construct a miniature model of supply and demand for the good (aided by an Excel sheet I provide). They then explain to their classmates what the equilibrium price and quantity would be and who would buy the good based on their data. Discussions that blend the material with activity away from the computer and allow some individual choice promote experiential learning and make discussions more engaging.

\section{(V)aried}

If students have multiple discussion questions per week, it is important to make sure the types of questions are varied. For example, if one question is primarily active (like the example above), this should be balanced with a question that is more reflective. This variation serves to both prevent discussions from being too repetitive as well as give students some flexibility in the type of questions they focus on first. Akin and Neal (2007) argue that variation in the types of discussions questions accommodates different student learning styles and backgrounds as well as allowing more time for discussion and reflection.

\section{(I)nteresting}

While it may seem like an obvious feature, a discussion question should be interesting. To make discussion questions interesting, I focus on topics that are relevant, personal and even controversial for students. I make questions topical and draw from recent politics, news and popular culture. For example, topics like minimum wage laws, unemployment rates affecting college graduates, student loan debt, gun control and national debt are topics with which most students have at least some familiarity and many may already have strong opinions. While there is always a chance of creating discussions that are "too heated," a "too heated" discussion is typically preferable to a boring one. Rossman (1999) finds that students prefer discussions that relate course material to their life or work situations. When students can directly relate to the topic of discussion they are likely to participate more enthusiastically as well as return and continue participate longer, creating a richer discussion.

\section{Open-ende(D)}

Finally, and perhaps most importantly, engaging discussion questions should be open-ended. While discussions should relate to and reinforce course material, they typically should not have a single "correct" answer (Velez-Solic, 2015). Nothing ends a discussion more quickly than a majority of students providing the same response or reaching the same conclusion. This can be especially challenging for more analytical subjects like economics or mathematics, where much of the course is focused on how to correctly solve a problem or apply a model. For example, a 
question like "If the minimum wage is raised, what does the supply and demand model predict will happen?" does not promote discussion. However, rephrased as "Do you personally think the minimum wage should be raised or not? Use the predictions of the supply and demand model to support your view" allows students to reach a variety of conclusions. A good discussion question is fundamentally different than an essay question. Students often enter a course from a wide variety of backgrounds and by choosing discussion questions that take advantage of differing views and provide an opportunity to relate these views back to course material makes discussions more engaging.

Besides the principles of the A.V.I.D. approach, some learning management system (LMS) tips I have found to be useful in promoting engaging discussions in $100 \%$ online courses include:

- Enabling a "users must post before seeing replies" feature. This prevents students from worrying that their response will be too similar to that of another student's. Rossman (1999) finds that students may experience guilt when not posting because other students have already posted a similar perspective. I would rather have repeated similar posts than students struggling to find something "new" to post.

- Disabling “allow students to edit and delete their own posts." This encourages students to be more careful with their wording/proof reading before posting. It also prevents students from posting and then deleting and reposting again. I include the following sentences in the syllabus to explain this policy: "Think of your posts as similar to saying something in a classroom. If you say something out loud you cannot delete or edit it after the fact (as much as we sometimes might want to!). If you wish to explain or expand on a post, then post a reply to your original post."

- Use a separate discussion for each discussion question. Trying to moderate and organize responses to different discussion questions in the same discussion rapidly becomes too difficult.

- Use threaded discussions. Threading allows better organization of replies, making it clearer when students are replying directly to each other.

\section{Managing Discussions and the Role of the Instructor}

Effectively moderating and grading discussion posts in an online course can be an intimidating and sometimes overwhelming job, especially in larger enrollment sections. In my typical class with around 50 students I will often have 200-300 posts per week to view and evaluate, many of which are made at unusual hours of the day. To help stay organized and avoid being overwhelmed, I separate my activity in the discussions into what I believe should be the three roles of an instructor in group discussions.

\section{Moderation}

The first role of an instructor in discussions is to moderate student posts. This means making sure that the discussion is progressing and on-topic. As a moderator it is not my role to respond to every student, but I will read and review every post. Rovai (2007) argues that in facilitating discussions, an instructor should avoid becoming the center or focus of a discussion and instead allow studentJournal of Teaching and Learning with Technology, Vol. 6, No. 1, January 2017 
to-student interaction to develop. I will respond to students who have posts without another response for a substantial amount of time. When I do respond to students I try to do it in a way that encourages further discussion, usually ending each response with a follow-up question or new perspective for them to consider. Asking follow-up probing questions and providing encouragement promotes continued discussion (Rovai, 2007). I also use moderation as an opportunity to make sure students remain on-topic and address the discussion question.

\section{Instruction}

One of the challenges in online discussions is that it is very easy for misunderstandings to be perpetuated if not caught early. When a student uses a term incorrectly or applies an idea in a flawed manner it can quickly create confusion or spread misinformation. My role in this case is to regularly review student posts to make sure they are not posting incorrect information. If they are applying a concept incorrectly, then I step in and provide clarification for them as well as the other students. I generally combine the roles of moderation and instruction when reviewing student posts. One learning management system tool that has been helpful in this regard is the option to “manually mark posts as read." Enabling this option allows me to mark as "read" posts I have viewed and do not need further attention while leaving as "unread" posts that I plan to return to, as well as those I have yet to read.

In addition to clarifying concepts, a second part of this role is to expand on course material in new ways. To this end, I generally respond to student posts when an opportunity presents itself to provide an alternative view, which often motivates further discussion, or else when a student posted a particularly interesting or unique view.

\section{Evaluation}

Finally, my third role in discussions is evaluation and grading of discussion posts. In my experience, if I am successful in keeping up in my other two roles (moderation and instruction) this can be fairly easy and is best done after the discussion deadline. By the discussion deadline, through the roles of moderation and instruction, I often have already completed many aspects of evaluation, such as making sure posts are on-topic and contribute to the discussion. This generally only leaves technical evaluation for things like length of posts, frequency of posts and distribution of posts, which are aspects that are much easier to evaluate independently from content.

Following the two sets of tips outlined in this article will help instructors design engaging discussion questions and effectively manage them in an online environment. The A.V.I.D. approach helps instructors write discussion questions that are more appealing to students and encourage greater and more active participation. Defining the role of the instructor into the three separate tasks of moderation, instruction and evaluation helps the instructor manage discussions without being overwhelmed. While designing and moderating discussion questions can be challenging, as online courses become increasingly popular with students as well as a larger part of many programs, promoting interaction and engagement through online discussions becomes crucial for effective instruction. 
Pollak

\section{References}

Akin, L., \& Neal, D. (2007). "CREST+ Model: Writing effective online discussion questions. Journal of Online Learning and Teaching, 3(2), 191-202.

Dillon, J. T. (1983). Teaching and the Art of Questioning. Bloomington, IN: Phi Delta Kappa Educational.

Herrington, J., Oliver, R., \& Reeves, T. C. (2002). Patterns of engagement in authentic online learning environments. ASCILITE 2002 Conference Proceedings, (pp. 279-286). Auckland, New Zealand.

Rossman, M. H. (1999). Successful online teaching using an asynchronous learner discussion forum. Journal of Asynchronous Learning Networks, 3(2), 91-97.

Rovai, A. P. (2007). Facilitating online discussions effectively. The Internet and Higher Education, 10(1), 77-88.

Velez-Solic, A. (2015). Teaching online without losing your mind: A comprehensive overview. Charleston, SC: AVS Academic Services.

Vygotsky, L. S. (1980). Mind in society: The development of higher psychological processes. Harvard University Press. 